\title{
Paradojas teóricas y estilísticas: geometría áu- rea en el hastial renacentista de la Catedral de León
}

\author{
César GARCÍA ÁLVAREZ \\ Univesidad de León
}

RESUMEN. Las proporciones del desmontado hastial occidental de la Catedral de León muestran que su diseño estuvo enteramente basado en el Número Áureo, y que su creador pensó el estilo gótico a través de procedimientos clasicistas propios del Renacimiento. Ello da lugar a algunas paradojas, la principal de las cuales es que, como los constructores originales del templo gótico usaron asimismo el Número de Oro como la base de su diseño, el hastial es, al mismo tiempo, aparentemente renacentista, pero estructuralmente es al mismo tiempo gótico y renacentista. Por el contrario, los restauradores de la Catedral consideraron el hastial antigótico y lo desmontaron para purificar la contaminada arquitectura del templo.

Palabras clave: Geometría áurea, proporción, arquitectura gótica, Renacimiento, catedral de León.

Abstract. The proportions of the dismonted Renaissance occidental hastial (gable end) of León's Cathedral show that its design was enterely based using the Golden Number, and that its creator thought the gothic forms through classical renaissance proceeds. This creates some paradoxes, the main of them is that, as the original constructors of the Gothic temple also used the Golden Number as the basis of its design, the hastial is at the same time apparently Renaissance, and structurally both Gothic and Renaissance. On the contrary, the restorers of the Cathedral considered the gable antigothic and desmonted it to purify the contamined architecture of the temple.

Key words: Golden Number, proportion, Gothic Architecture, Renaissance, Cathedral of Leon.

La historia constructiva de los diferentes hastiales que han coronado la fachada occidental de la catedral de León encierra una serie de aspectos sorprendentes que originan inesperadas e irónicas paradojas teóricas, metodológicas y estéticas. Éstas sólo pueden ser desveladas mediante el análisis e interpretación tanto de los fundamentos geométricos del hastial renacentista como de la comprensión de éstos por parte de los arquitectos decimonónicos que restauraron el templo leonés. Este artículo pretende desvelar dichas paradojas.

Desde el siglo XIII, tres han sido los hastiales que han coronado la fachada occidental de la catedral de León. El actual, obra de Demetrio de los Ríos, uno de los artífices de la restauración integral de la catedral en las últimas décadas del siglo 
XIX, sustituyó al hastial renacentista, que constituye el objeto de nuestro estudio, y que a su vez reemplazó a otro anterior, medieval. De los dos primeros poseemos datos precisos que permiten efectuar estudios fiables y no simples especulaciones. Poco se puede afirmar, sin embargo, del hastial medieval. Su existencia parece probada por algunos testimonios pictóricos conservados. Javier Rivera ha mostrado las posibles referencias de carácter gráfico del remate occidental en tres obras pictóricas de finales del siglo $\mathrm{XV}$ y principios del siglo $\mathrm{XVI}^{1}$. La primera, atribuida al llamado maestro de Palanquinos, de mediados del siglo XV; la segunda, una Presentación de Jesús en el Templo, de la colección Simonsen de Saô Paulo, y una tercera anónima, del segundo cuarto del siglo XVI, quizá de la escuela de Juan de Borgoña. En opinión de Rivera, es probable que el hastial se hubiese desmontado en las primeras décadas del siglo XVI, por causas desconocidas, $\mathrm{y}$ que se encargase su sustitución al maestro de obras activo en aquel momento.

Sea como fuere, lo cierto es que no disponemos de documentos ni testimonios gráficos lo suficientemente precisos que nos permitan reconstruir aceptablemente la forma, dimensiones y estructura del hipotético hastial medieval. Bien a causa de un derrumbe, bien porque realmente no llegó a finalizarse, en el siglo XVI se procedió a erigir un hastial realizado en un estilo aparentemente contradictorio con el del resto del edificio, una construcción que en el purista e historicista siglo XIX fue considerado formal y estéticamente irreconciliable con el conjunto de la fábrica gótica.

La construcción del hastial renacentista prolonga el ambicioso programa artístico efectuado en el templo catedralicio

\footnotetext{
${ }^{1}$ J. RIVERA, Historia de las restauraciones de la Catedral de León "PULCHRA LEONINA": La contradicción ensimismada, Valladolid, 1993.
}

durante el obispado de D. Pedro Manuel. En los años de su prelatura se llevaron a cabo actuaciones importantes como la finalización del claustro, la crestería, las torrecillas del costado norte de la iglesia, la escalera capitular, el trascoro, los retablos, los sepulcros de San Alvito y el obispo D. Pelayo, la capilla de Santa Catalina y el citado remate del hastial occidental, al que dedicaremos toda nuestra atención.

La autoría del hastial renacentista no está todavía totalmente aclarada. Según Javier Rivera, la coronación del imafronte fue proyectada hacia el año 1537 por Juan de Badajoz el Mozo, y concluida en 1567 por el maestro Juan de Rojas ${ }^{2}$. Se conservan diversos grabados y fotografías ${ }^{3}$, anteriores a la restauración y desarticulación efectuada a finales del siglo XIX, que documentan su disposición y su configuración formal (véanse las figuras 1-3). En ellas se aprecian las características formales y la composición del remate. Sobre el rosetón correspondiente a la nave central se superponía un primer cuerpo con el relieve de la Anunciación. Sobre él, un segundo cuerpo, formado por una peineta calada y rematada de forma triangular, y ornamentada con relieves. En los dos extremos del frontispicio triangular, se levantaban dos torres unidas a éste por tirantes oblicuos, con dos chapiteles de forma helicoidal, coronados por las estatuas de dos hombrecillos desnudos (véanse las figuras 1, 2, 3 y 7).

\footnotetext{
2 Ibídem.

${ }^{3}$ Tenemos conocimiento del primitivo imafronte del siglo XVI a través de varios grabados y algunas fotografías anteriores a la restauración. Figuran entre los grabados que reproducen el hastial el publicado por A. PONZ en su Viaje de España (Tomo XI, Madrid, 1787, pág. 200). Son destacables las reproducciones de J. HOAG, en Rodrigo Gil de Hontañón. Gótico y Renacimiento en la arquitectura española del siglo XVI, Madrid, 1985, pág. 207. Asimismo, J. RIVERA, op. cit., pág. 64.
} 
Demetrio de los Ríos, en su monografía sobre la catedral de León ${ }^{4}$, atribuyó la autoría de este hastial a los Badajoz, padre e hijo, aunque en la actualidad diversos estudios han puesto en tela de juicio la cronología y adscripción a estos arquitectos sostenidas por de los Ríos. Javier Rivera ${ }^{5}$ y Gloria C. Carrizo ${ }^{6}$ afirman que Juan de Badajoz el Mozo aportó posiblemente unas trazas hacia el 1537, anulando por tanto la intervención de Juan de Badajoz el Viejo. Estas trazas serían visibles en el desarrollo helicoidal de los chapiteles. Según Rivera, la decoración de las espirales encierra un carácter simbólico que estaría en consonancia con las ideas propias de la segunda mitad del siglo. Por otra parte, la utilización de las columnas jónicas que enmarcan la roseta, los motivos decorativos del friso, y los florones superiores, son rasgos estilísticos igualmente más acordes con un período cronológico posterior a la época creativa de Juan de Badajoz el Mozo, e invitan a pensar que la construcción del hastial se prolongaría años después del fallecimiento de éste en 1554, hasta ser coronado bajo la dirección de Juan López de Rojas, quien habría finalizado el hastial hacia al año 1567, haciéndose cargo de la decoración escultórica Bautista Vázquez.

Otros autores, como Gómez Moreno, sostienen que las trazas fueron realizadas por Juan López, y retrasan consecuentemente la fecha de construcción hasta 1570. Por su parte, J. Hoag afirma que las trazas del imafronte fueron realizadas por Rodrigo Gil de Hontañón en el año 1558, aunque la intervención se efectuó varios años des-

\section{-}

${ }^{4}$ D. DE LOS Ríos y SERRANO, La Catedral de León (Monografía), Tomo I, págs. 37, 1895.

${ }^{5}$ Sobre el hastial renacentista, véase también J. RIVERA, Arquitectura en León en la segunda mitad del siglo XVI, León, 1982, pp. 63-66.

${ }^{6}$ G. C. CARRIZO CASADO, La escultura del siglo XVI en León. Tesis doctoral inédita. Universidad Complutense de Madrid, 1988. pués por otros maestros del templo leonés que respetaron los planteamientos del autor del Palacio de los Guzmanes. En la documentación del archivo catedralicio no se conservan referencias a esta obra que puedan proporcionarnos alguna pista sobre su concreción tanto cronológica como artística. En opinión de Ma . Dolores Campos Sánchez-Bordona ${ }^{7}$, lo más lógico sería atribuir la ejecución al maestro Juan López de Rojas, arquitecto fuertemente influenciado por las formas artísticas de Juan de Badajoz el Mozo, y que sería el responsable de la dirección de la fábrica catedralicia en torno a las décadas de 1560-1570.

El hastial renacentista fue desmontado por Demetrio de los Ríos durante la restauración del templo a finales del siglo XIX. Ignacio González-Varas ha analizado con profundidad el proceso de sustitución del hastial, como parte de un proyecto de purificación estilística que recuperase la unidad gótica primigenia que los avatares de la historia habían desvirtuado ${ }^{8}$. Para justificar su intervención, Demetrio de los Ríos adujo la inestabilidad de la obra, los riesgos que comportaba para la sostenibilidad del conjunto del edificio, y siete "defectos de construcción" (fundación de las pilas de caracol fuera de correspondencia con el macizo de la parte más expuesta; desequilibrios en la unión del hastial con las torrecillas; piedra de pésima calidad; incorrecta restauración ya en el siglo XVI, a cargo de Juan de Badajoz el Mozo, cuerpos volados fuera del paramento exterior del hastial; desconexión entre sus muros y los del interior del templo, y excesiva elevación del hastial $)^{9}$. Todos estos defectos refrendaban en opinión de Demetrio de los Ríos que el

\footnotetext{
${ }^{7}$ M. D. CAMPOS SÁNCHEZ-BORDONA, Juan de Badajoz y la arquitectura del Renacimiento en León, León, 1993.

${ }^{8}$ D. DE LOS RíOS, op. cit., p. 378. I. GONZÁLEZVARAS, La Catedral de León, historia y restauración, León, 1993

${ }^{9}$ Ibídem, p. 382.
} 
hastial renacentista había sido levantado en contradicción con los principios constructivos del estilo gótico, como un objeto ornamental destinado a satisfacer la vista del público, pero con total desprecio de las verdades de la arquitectura gótica. Otros autores, como José Caveda y Nava, a pesar de apreciar el valor estético y la belleza del hastial, afirmaban igualmente que en él no había "mezcla alguna de gótico" ${ }^{10}$. Como veremos, la verdad resulta ser doblemente contradictoria con estas opiniones decimonónicas, y origina inesperadas paradojas estilísticas y teóricas.

La pérdida de partes del hastial renacentista y el estado fragmentario de los restos conservados impide efectuar mediciones precisas, pero es posible deducir sus proporciones relativas a partir de las fotografías y dibujos que muestran su apariencia original. Contamos con cuatro documentos principales. En primer lugar, la fotografía que mejor ha transmitido el aspecto de la antigua fachada, (figura 1), la ortografía realizada por Manuel Navarro y Fernando Sánchez Pertejo en 1794 (figura 2), el alzado de la fachada dibujado por Ricardo Velázquez Bosco (figura 3), y el minucioso dibujo elaborado por Demetrio de los Ríos (figura 7). La fotografía confirma la corrección de las proporciones fijadas por los tres dibujos, y especialmente el de Velázquez Bosco ${ }^{11}$. A partir de ambos testimonios gráficos es posible elaborar la siguiente tabla de proporciones (expresadas en centímetros, y que se corresponden con las dimensiones del dibujo de Velázquez

${ }^{10} \mathrm{~J}$. CAVEDA, Ensayo histórico sobre los diversos géneros de arquitectura empleados en España desde la dominación romana hasta nuestros días, Madrid, 1848, pp. 355356, citado por I. GONZÁLEZ VARAS, op. cit., p. 388.

${ }^{11}$ Dado que el dibujo de Demetrio de los Ríos sólo representa el cuerpo del hastial destinado a ser sustituido, sólo es posible deducir a partir de él las proporciones del hastial, pero no su relación proporcional con el resto del imafronte gótico. Aún así, sus proporciones confirman las de los dos restantes dibujos.
$\left.\operatorname{Bosco}^{12}\right)$.

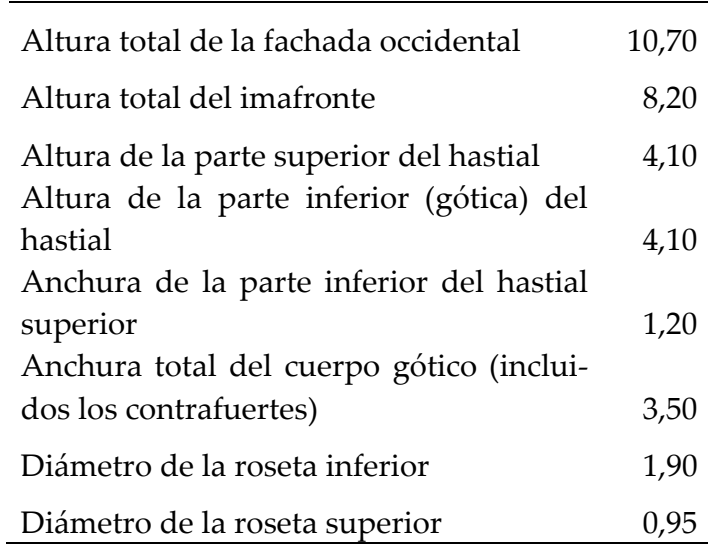

Como puede comprobarse, no hemos incluido la totalidad de las medidas, y sólo ofrecemos una selección de las que consideramos más importantes para lograr comprender el propósito estético del creador del hastial. Para unarquitecto renacentista, las medidas absolutas no poseen una importancia decisiva, lo esencial es el conjunto de relaciones proporcionales existentes entre las diversas partes del edificio. Para conocer qué dimensiones del hastial son las verdaderamente importantes, es necesario intentar comprender los motivos y el propósito que guiaron a su autor, y reconstruir el itinerario de su pensamiento. Es posible suponer que, hacia 1530, el estado del imafronte estuviese incompleto, fuera cual fuera la causa de tal estado (ruina o inexistencia del hastial). De ser así, lo que el arquitecto podía observar, y considerar como imafronte, era una realidad doble. Por un lado, el conjunto de la fachada gótica. Si se acepta, como parece lógico, que el hastial renacentista empezaba justo donde terminaba la obra gótica, entonces la altura total de la fachada gótica se extendía desde la base del parteluz del arco central (figuras 4, 5 y 6, punto 0) hasta el punto en el que se interrumpía la fábrica (B-B, 6'6 centíme-

\footnotetext{
${ }^{12}$ Las dimensiones absolutas pueden quedar alteradas, obviamente, en su reproducción gráfica para el presente artículo.
} 
tros). Por otro lado, sobre el cuerpo inferior que contenía los tres arcos del ingreso al templo, se apoyaba un cuerpo superior del imafronte, separado de las torres laterales y sólo ligado a ellas por arbotantes, (A-A'-B$B^{\prime}, 3^{\prime} 5$ de anchura). Fue en esta línea B-B' donde se produjo exactamente el corte final entre la obra medieval y la renacentista. Ésta era la realidad de la que tuvo que partir el arquitecto renacentista. Para comenzar su proyecto, el primer paso consistía necesariamente en delimitar tanto la altura total del nuevo hastial como el espacio en el que podían perfilarse sus formas. Para lograr solucionar este problema, el arquitecto procedió a multiplicar la altura total del cuerpo conservado (0-0'), por el número áureo. El resultado $(6,6 \times 1,618=10,67)$, se corresponde exactamente con la altura de la estatua que coronaría el hastial $\left(\left(0-0^{\prime}\right) \Phi=0-0^{\prime \prime}\right)$. De este modo, el número áureo enlaza la altura total resultante con la altura original del conjunto.

Para definir de un modo más preciso el espacio que iba a ocupar la nueva obra, se partió del rectángulo formado por el cuerpo superior del imafronte. La altura de este rectángulo originario (figuras 4 y 5 , A$\mathrm{A}^{\prime} \mathrm{B}-\mathrm{B}^{\prime}$ ) era de 4,1 (siempre en cms. relativos al dibujo de Velázquez Bosco), su anchura total 3,5, y su anchura interior 2,2. Por encima de éste rectángulo, el arquitecto renacentista proyectó en altura otro rectángulo idéntico (B-B'-C-C'), que resulta ser coincidente con la prolongación áurea de la altura originaria. De este modo, el rectángulo del cuerpo superior de la fachada gótica se reduplicó para acoger el futuro hastial. Ambos rectángulos, idénticos, creaban una totalidad que mantenía una relación dupla (2/1) con sus partes. Esta proporción dupla es profundamente acorde con la sensibilidad renacentista. Se corresponde con la proporción musical del intervalo de octava (2/1) y origina un sistema de correspondencias simple, armónico, y matemáticamente perfecto. Como muestran los dibujos, el nuevo rectángulo acogería el conjunto del nuevo hastial, incluida la estatua que lo coronaba. La parte gótica y la renacentista pasaban a ser así dos rectángulos de idénticas dimensiones. Al mismo tiempo, esta relación dupla reforzaba la relación áurea existente entre la altura del nuevo hastial, la de la fachada gótica originaria, y la nueva altura total de la fachada ${ }^{13}$.

Sin embargo, resulta evidente que esta línea de separación estilística no diferencia totalmente el imafronte del hastial propiamente dicho. El arquitecto decidió prolongar el macizo del paramento gótico hasta una altura más elevada. Ahora bien, si dividimos la altura total del rectángulo duplo $(8,2)$ por la altura a la que el arquitecto emplazó el límite del muro (5), el resultado es 1,618, de nuevo la proporción áurea. Ello significa que el arquitecto procedió a dividir la altura total del nuevo rectángulo duplo por el número áureo (A-C / $\phi=\mathrm{D}^{\mathrm{D}} \mathrm{D}^{\prime}$; $8,2 / 1,618=5,068$ ). La mitad de la altura del nuevo paramento macizo resultante (A-D / 2), coincidía además de un modo casi exacto con el centro del rosetón gótico.

La utilización del número áureo en el hastial no se limita sin embargo a estos aspectos reseñados. Mediante el trazado de las subdivisiones áureas de las diferentes alturas y de los rectángulos duplos, el arquitecto determinó la altura total del nuevo imafronte y el espacio que debía ocupar el cuerpo superior del nuevo hastial. Para poder precisar algunas dimensiones del mismo se sirvió nuevamente de la divina proporción. Así se comprueba al multiplicar por 1,618 la altura del rectángulo del ima-

\footnotetext{
13 Asimismo, la línea A-A', que marca la separación entre el cuerpo inferior y el superior del imafronte gótico, corta al eje central 0-0' exactamente en su sección áurea. Consecuentemente, el cuerpo superior del imafronte gótico, que alberga el rosetón, mantiene una relación dupla con el rectángulo superior del nuevo hastial $\left(B-B^{\prime}-C-C^{\prime}\right)$, y áurea con el rectángulo inferior $\left(\mathrm{A}-\mathrm{A}^{\prime}-\mathrm{I}-\mathrm{I}^{\prime}\right)$.
} 
fronte gótico (A-B), cuyo resultado delimita lo que sería el friso del cuerpo inferior del hastial (E-E'), línea que coincide además con el centro del cuadrado C'-D-D', con lo que de nuevo se combinan las proporciones duplas y las áureas. Asimismo, para determinar la línea inferior del cuerpo que acoge a la roseta superior $\left(\mathrm{F}-\mathrm{F}^{\prime}\right)$, multiplicó por la proporción áurea la altura entre la base de la fachada y el centro del rosetón inferior $((0-\mathrm{x}) \Phi=\mathrm{I}-\mathrm{F})$ En el centro del espacio rectangular inferior (D-D'-E-E'), situó a su vez el centro del rosetón de dicho cuerpo, atravesado por la línea G-G', que delimita al mismo tiempo el arranque de los remates de los pináculos y el punto de apoyo de los arbotantes que unen el cuerpo central del hastial con dichos pináculos. El remate triangular del hastial pasó consiguientemente a ocupar, junto con el friso de unión entre ambos cuerpos, el rectángulo superior C-C'-E-E'.

El dibujo de Demetrio de los Ríos (figura 7) nos permite profundizar en las relaciones proporcionales existentes entre las partes interiores del hastial propiamente dicho. Dado que sólo reproduce la obra renacentista, no resulta posible analizar a partir de él sus relaciones con la fachada gótica. A la hora de diseñar las partes concretas del hastial, el arquitecto renacentista se sirvió de nuevo fundamentalmente de proporciones duplas y áureas. Así, la altura total del cuerpo renacentista (A-B) encuentra su punto medio exactamente en el centro de la roseta del hastial (C). La base de la balaustrada coincide con la mitad de la distancia entre el centro de la roseta y la base del conjunto $((\mathrm{A}-\mathrm{C}) / 2=\mathrm{D})$. En el centro de la distancia entre este punto (D) y el coronamiento del hastial (B), se encuentra el límite inferior del friso del hastial (E). Estos puntos proporcionales son coincidentes, por lo demás, con las líneas áureas y duplas generadas en el conjunto de la fachada. El punto A se corresponde con $0^{\prime}, \mathrm{D}$ está en el centro de la línea D-D', C en el centro de la línea G-G', y E en el centro de la línea E-E'.

Asimismo, el radio de la roseta superior equivale a la novena parte de la altura total del cuerpo superior del hastial (A-B / 9 $=\mathrm{I}-\mathrm{I}^{\prime}$ ). Por otra parte, los puntos H y G, que marcan con notable precisión la parte superior e inferior del escudo de la ciudad, resultan de dividir la distancia entre el centro de la roseta y la cabeza de la estatua superior por el número áureo $((\mathrm{B}-\mathrm{C}) / \Phi=\mathrm{H}$ ó $\mathrm{G})$, que de este modo también está presente en el trazado proporcional del cuerpo superior del hastial, combinado con las proporciones duplas.

Las secciones del hastial en anchura son igualmente sencillas de definir, y se basan también en relaciones duplas. La anchura total del centro del hastial ((C-X) $\mathrm{x}$ 2), es el doble de la anchura de cada pináculo (Y-Z), que a su vez es el doble del espacio vacío intermedio entre ambos cuerpos (X$Y)$. El radio de la roseta superior es además, como hemos visto, la novena parte de la altura total de la nueva obra renacentista (A-B / 9). Al multiplicar el diámetro de la roseta por el número áureo, resulta la mitad de la anchura total del hastial (C-Z). Las relaciones no pueden ser más sencillas, y se completan con el hecho de que el diámetro de la roseta superior sea exactamente la mitad del diámetro del rosetón gótico, como se puede deducir de los tres testimonios gráficos (figuras 4,5 y 6).

Mediante esta combinación de proporciones duplas y áureas, de explicación algo abstrusa pero de trazado sencillo, la estructura esencial del hastial y sus relaciones proporcionales fundamentales quedaban perfectamente trazadas. Desde luego, estas suposiciones pueden ser erróneas, porque nos vemos obligados a basarnos en dibujos y fotografías y no en la observación y medición de la obra original, pero creemos que su concordancia con los documen- 
tos gráficos que conservamos avala su corrección. El procedimiento geométrico utilizado demuestra que el arquitecto siguió principios puramente renacentistas, basados en proporciones musicales y áureas nacidas de números sencillos, según el ideal de concinnitas definido por Alberti ${ }^{14}$. No vamos a descubrir en este artículo la importancia de las proporciones áureas para el pensamiento estético y la creación artística del Renacimiento, al ser sobradamente conocida y constituir casi un tópico historiográfico $^{15}$. El número áureo está aquí empleado como el mejor recurso posible para lograr una armonía homotética entre diferentes magnitudes ${ }^{16}$. El diseñador renacentista del hastial combinó la relación dupla y áurea de las diferentes dimensiones de la fachada, estableciendo relaciones proporcionales sencillas, armónicas y musicales.

Ahora bien, si en el trazado básico de las proporciones del hastial el arquitecto mantuvo una fiel observancia de la geometría áurea, resultan cuando menos sorprendentes a primera vista las formas finalmente elegidas para el trazado del cuerpo superior del hastial. Lo que el arquitecto creó fue una reproducción del mismo esquema de la totalidad de la fachada gótica original.

En efecto, el cuerpo superior del hastial es una reproducción, reinterpretada, de

${ }^{15}$ Son conocidas las objeciones de Rudolf Wittkower a la utilización de las proporciones basadas en el número áureo por parte de los arquitectos renacentistas. Se trata de un problema complejo, que el historiador germano aplicó sobre todo a la arquitectura italiana. De todos modos, cuando las proporciones de los edificios revelan con exactitud la presencia de proporciones áureas, parece que lo más prudente es dejar de lado los apriorismos teóricos, y atenerse a la realidad de la obra. Cfr. el fundamental estudio de R. WITTKOWER, Los fundamentos de la arquitectura en la Edad del Humanismo, Madrid, 2007.

${ }^{16}$ Para la comprensión de las proporciones y el número áureo, véase el fundamental estudio de M. C. GHYKA, El número de oro, Barcelona, 1986.
}

todo el conjunto de la fachada occidental de la catedral leonesa. Las dos torretas laterales que flanquean el cuerpo central son así equivalentes a las dos grandes torres que delimitan la fachada, y el rectángulo central, con la roseta y el coronamiento en forma de triángulo, equivale al cuerpo central del imafronte, a su parte inferior. Aún existe otro aspecto más, muy significativo. Una de las peculiaridades de la Catedral de León que más ha llamado la atención de los historiadores y arquitectos es que el cuerpo central del imafronte no está unido a las torres laterales, sino que se separa de ellas, y permanece ligado únicamente por medio de arbotantes, lo que le confiere a la fachada un aire ligero y esbelto, y acentúa su trasparencia, su carácter gótico. Pues bien, es muy posible que este aspecto llamase también la atención del arquitecto renacentista, porque ha repetido la misma solución en el remate del hastial. El cuerpo central se une a las dos torretas laterales mediante una especie de arbotantes. La forma de éstos, que sólo se puede apreciar a partir del dibujo de Demetrio de los Ríos (figura 7), no es sin embargo exactamente la de un arbotante gótico, ya que su parte inferior es más bien una voluta. Ello significa que el autor del hastial mezcló elementos góticos $\mathrm{y}$ renacentistas, y por ello funde un arbotante con una voluta. Las volutas desempeñaban en el estilo renacentista, desde la obra de Alberti, la función de suavizar visualmente la transición y la comunicación entre los dos cuerpos rectangulares en los que se dividían las fachadas (como en Santa María Novella, en Florencia) ${ }^{17}$. En el hastial, la voluta comunica el cuerpo central con las torretas laterales. Desempeña, por tanto, una función muy similar, que fusiona de un modo sorprendente $\mathrm{y}$ original formas $\mathrm{y}$ funciones góticas y renacentistas.

\footnotetext{
${ }^{17}$ Véase el análisis de G. C. ARGAN, Renacimiento y barroco, vol. I, Barcelona, 1985, sobre el significado de las volutas en Alberti.
} 
La repetición del cuerpo inferior de una fachada en el cuerpo superior de la misma, con leves alteraciones y con las obvias diferencias de magnitud, es muy frecuente en el primer Renacimiento hispano $^{18}$, hasta el punto de constituir una de sus señas formales de identidad. Fachadas como las del Hospital de Santa Cruz y el Alcázar de Toledo, la antigua Universidad de Burgo de Osma, el convento de las Dueñas de Salamanca, San Cecilio, Santa Ana, y San Matías de Granada, la catedral de Almería, Santa María de Calatayud, el Ayuntamiento de Uncastillo, entre otras, así lo demuestran. En ocasiones, la repetición de tal esquema sirve para efectuar una superposición de órdenes típica del estilo (dórico, jónico y corintio es la fórmula más usual), siguiendo el ejemplo del Coliseo romano. Algo parecido pudo intentar el arquitecto catedralicio, porque las columnas del hastial superior son de estilo jónico, como si tuvieran que corresponderse con otras dóricas en el piso inferior.

El hastial renacentista permite por todo ello reflexionar sobre el problema de las relaciones entre el estilo gótico y el clasicismo renacentista a la italiana. En mi opinión, el aspecto más significativo del hastial reside en el hecho de que un arquitecto que se ha formado en el nuevo estilo renacentista se vea obligado a completar una edificación previa, realizada en estilo gótico. Por ello, en el hastial se reproduce el problema de las relaciones entre lo que la historiografía ha denominado el estilo "moderno" y el "romano" ${ }^{19}$. En este conflicto puede hallarse la clave de la obra. No es aventurado

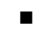

${ }^{18}$ Tradicionalmente denominado "plateresco". No vamos a entrar en este momento en la disputa terminológica que ha acompañado al problema de la definición estilística de los inicios de la arquitectura española de finales del siglo XV y principios del siglo XVI.

${ }^{19}$ Cfr. V. Nieto, A. J. Morales, F. ChecA, Arquitectura del Renacimiento en España (1488-1599), Madrid, 1989. imaginar el posible desconcierto que hubo de tener el arquitecto antes de comenzar la obra. Tenía que respetar una edificación de un estilo contrario al de su formación. Estaba obligado a ello, y al mismo tiempo sentiría la necesidad de expresarse con un lenguaje arquitectónico distinto. La solución que ofreció no sólo fue acertada y coherente con el estilo de su época, sino profundamente original. El autor pensó la fachada gótica como si fuera renacentista, y la completó siguiendo una estructura geométrica al modo romano, clásica, pero finalizada con formas que mezclaban visual y estructuralmente el gótico y el nuevo estilo, al modo moderno. Gótico moderno y Renacimiento clasicista a lo romano se funden así de un modo llamativo e inesperado, y la fachada se puede contemplar tanto como una obra gótica con un remate renacentista, cuanto como una fachada renacentista elaborada con formas góticas.

Lo que resulta sumamente llamativo, y da lugar a una de las paradojas que anunciábamos en la introducción, es que el diseñador del hastial haya sido tan fiel a la apariencia y estructura de las formas góticas. La paradoja no reside sólo en este respeto por parte de un arquitecto renacentista hacia elementos del estilo gótico, ya de por sí reseñable, sino sobre todo en que dichas formas, reinterpretadas a lo moderno, se superpongan a una estructura geométrica pensada al romano. Que un esquema geométrico renacentista basado en el número áureo acoja unas formas visual y estructuralmente góticas es en apariencia una contradicción. Pero sólo en apariencia, y aquí se produce una segunda paradoja, que reside en el hecho de que los arquitectos góticos, como se aprecia en la misma catedral de León, no sólo no desconocieron el número áureo y sus propiedades y aplicaciones constructivas, sino que se sirvieron de él de un modo sumamente frecuente e intenso 
que sólo la historiografía de las últimas décadas ha llegado a revelar ${ }^{20}$. Como hemos expuesto en otro texto, y como otros estudiosos han igualmente mostrado, la planta de la catedral gótica leonesa fue trazada de acuerdo con estructuras y tramas geométricas puramente áureas ${ }^{21}$. En las figuras 8 y 9 se aprecia la correspondencia de la planta con un sistema de rectángulos duplos combinados con rectángulos áureos, exactamente el mismo procedimiento empleado por el arquitecto renacentista que diseñó el hastial.

Llegados a este punto, la paradoja adquiere tintes irónicos e incluso cómicos. Por un lado, según la opinión renacentista sobre el arte de los siglos medievales, fijada por Vasari y asumida por humanistas y artistas, la arquitectura medieval se había caracterizado por un olvido trágico de los elevados conocimientos de la sacrosanta Antigüedad grecorromana. Los arquitectos góticos eran a ojos renacentistas unos bárbaros ignorantes. Los heroicos artistas y humanistas del Renacimiento habían devuelto el esplendor artístico perdido, fundamentándolo sobre sólidas bases científicas, entre las que sobresalía la divina proporción, el número áureo. Pero ahora sabemos que los arquitectos góticos no eran tan ignorantes al fin y al cabo, y habían

\footnotetext{
${ }^{20}$ Los estudios fundamentales que han desvelado la trascendencia de la geometría áurea para la arquitectura gótica son la obra de F. M. LUND, Ad Quadratum, I y II, MOESSEL, Die Proportion in der Antike und Mittelalter, M. GHYKA, Estética de las proporciones en la naturaleza y las artes, Barcelona, 1988, El número áureo, op. cit., y Filosofía y mística del número, Barcelona, 1999, así como la obra de J. HANI, El simbolismo del templo cristiano, Sophia Perennis, Palma de Mallorca, 1997.

${ }^{21}$ C. GARCÍA ÁLVAREZ, «Geometría y proporciones áureas en la planta de la Catedral de León», Boletín del Instituto Camón Aznar, no 84, 2001, pp. 49-80. J. M. MERINO DE CÁCERES, «Metrología y simetría en las Catedrales de Castilla y León» en P. NAVASCUÉS PALACIO y J. L. GUTIÉRREZ ROBLEDO (eds.), Medievalismo y neomedievalismo en la arquitectura española: Las Catedrales de Castilla y León I, Ávila, 1994, pp. 9-52.
}

trazado sus edificios de acuerdo a aquellas fórmulas y principios que constituían el corazón de la geometría antigua. Como desconocemos la formación intelectual del arquitecto que trazó el hastial leonés, no podemos en justicia generalizar y atribuirle un desprecio similar al vasariano hacia las formas creadas por los bárbaros góticos. La repetición en el hastial del audaz sistema de arbotantes de la fachada invita a pensar que pudo incluso sentir admiración por los logros del edificio. Con lo que no contaba el arquitecto renacentista era con la paradójica interpretación que tanto del hastial como de la arquitectura gótica iba a efectuar un enamorado del gótico como Demetrio de los Ríos. Con toda probabilidad, el arquitecto decimonónico no llegó a sospechar que su amada arquitectura gótica se fundamentaba en un principio tan clásico como la geometría áurea. Pero mucho menos aún pudo imaginar que el hastial lleno de defectos de construcción, que según él afeaba el conjunto del templo y se situaba en abierta contradicción con sus principios constructivos, respetaba no sólo las formas y las estructuras de la fachada y de todo el estilo, sino el mismo fundamento geométrico que había servido para edificar la catedral. Lo más llamativo de toda esta involuntaria paradoja es que el arquitecto renacentista prolongó el espíritu gótico, quizá inconscientemente, pero en cualquier caso de un modo mucho más fiel a los principios geométricos góticos que el apasionado y romántico amante decimonónico. Éste le acusó de un crimen que no había cometido, y que sólo existía desde la particular atalaya ideológica que le llevaba a defender la pureza de un estilo gótico al que creía conocer verdadera y científicamente, pero del que desconocía uno de sus principios esenciales, compartido por la obra que tanto defenestró por considerarla antigótica.

Si Demetrio de los Ríos hubiera advertido esta profunda afinidad entre el hastial que quería derribar y el templo góti- 
co al que en realidad estaba rindiendo un paradójico homenaje, quizá habría desistido de su empeño purificador, y no lo habría sustituido por el sin embargo hermoso hastial neogótico que hoy en día remata la fachada occidental. Porque lo más paradójico de todo es que el hastial de Demetrio de los Ríos, al no servirse de la geometría áurea, resulta al final ser menos gótico que el hastial renacentista, "defectuoso y bramantesco". Fue más fiel el supuesto traidor que el ardiente vasallo. La historia del arte es, a veces, profundamente irónica.

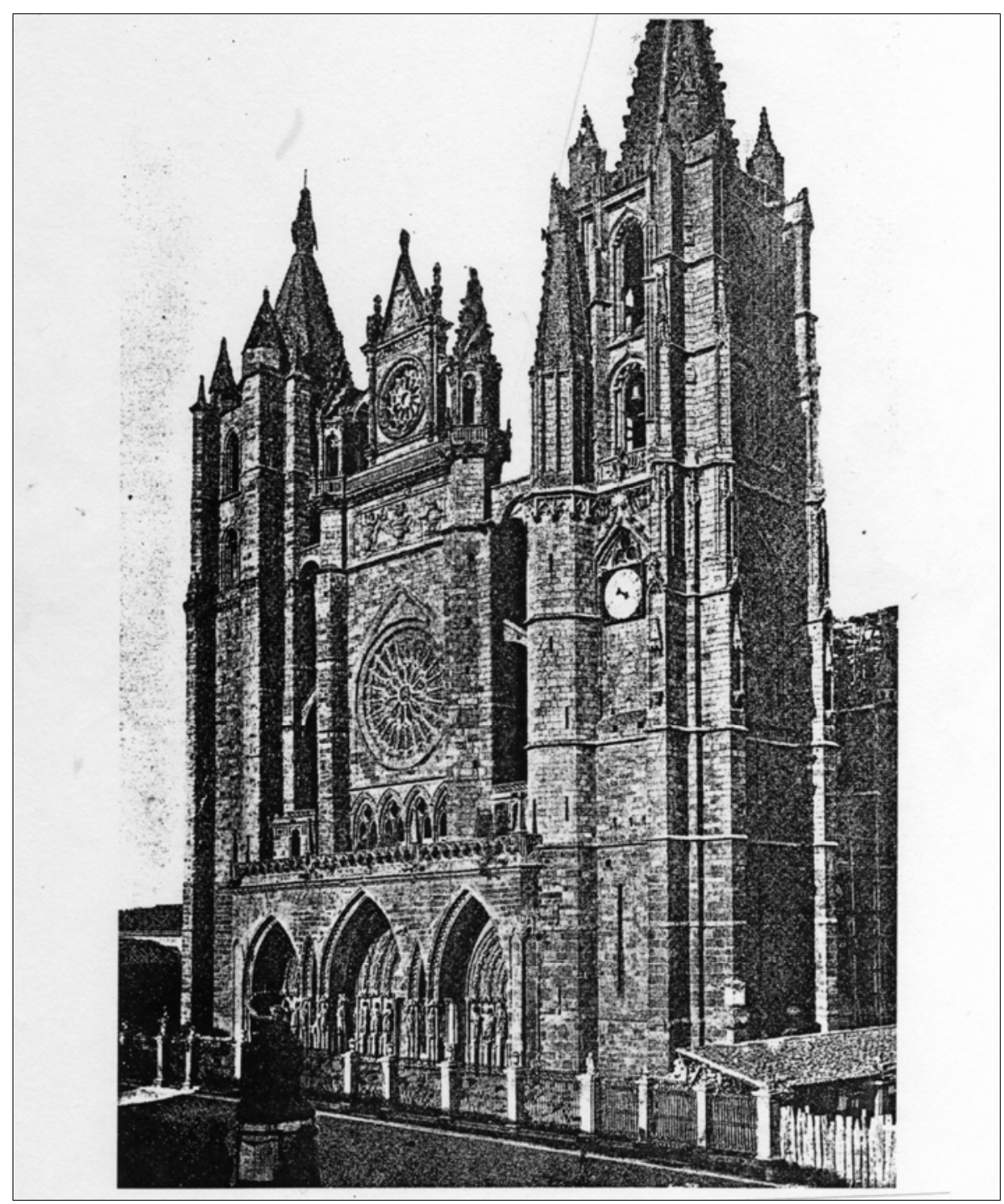

- Fig. 1. Fotografía de la fachada occidental de la Catedral de León antes de la Restauración decimonónica. 


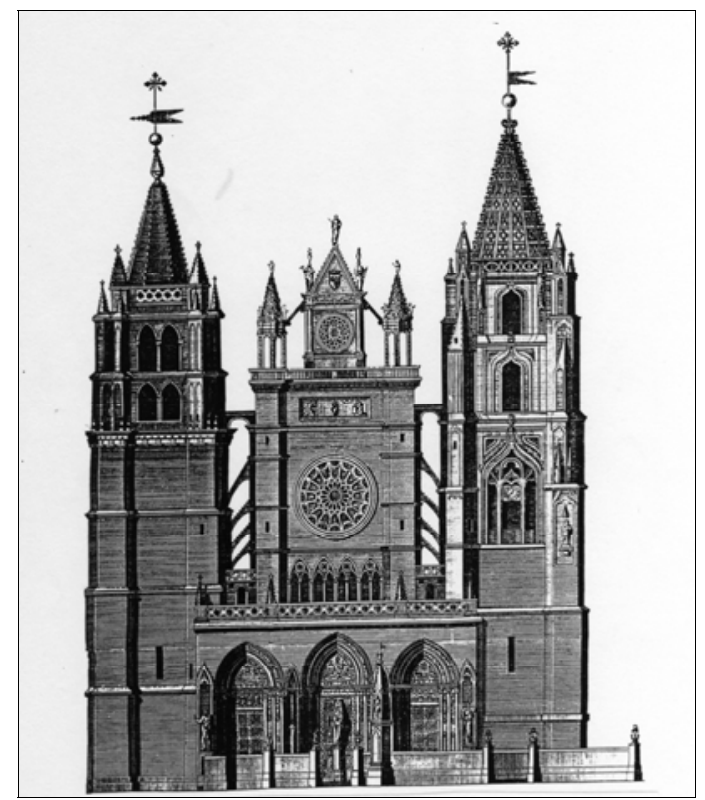

- Fig. 2. Ortografía de la fachada occidental de la Catedral de León, de Manuel Navarro y Fernando Sánchez Pertejo (1794).

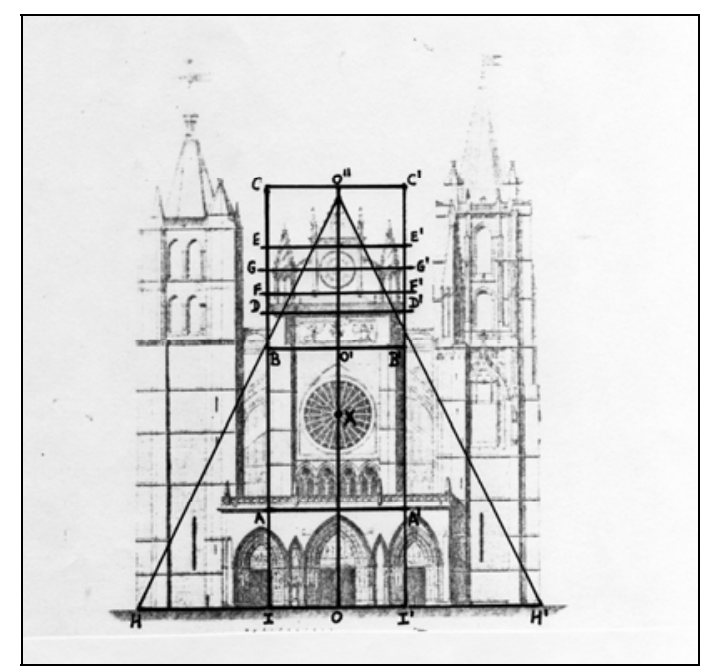

- Fig. 4. Trazado del diseño proporcional del hastial renacentista, sobre el alzado de Velázquez Bosco.

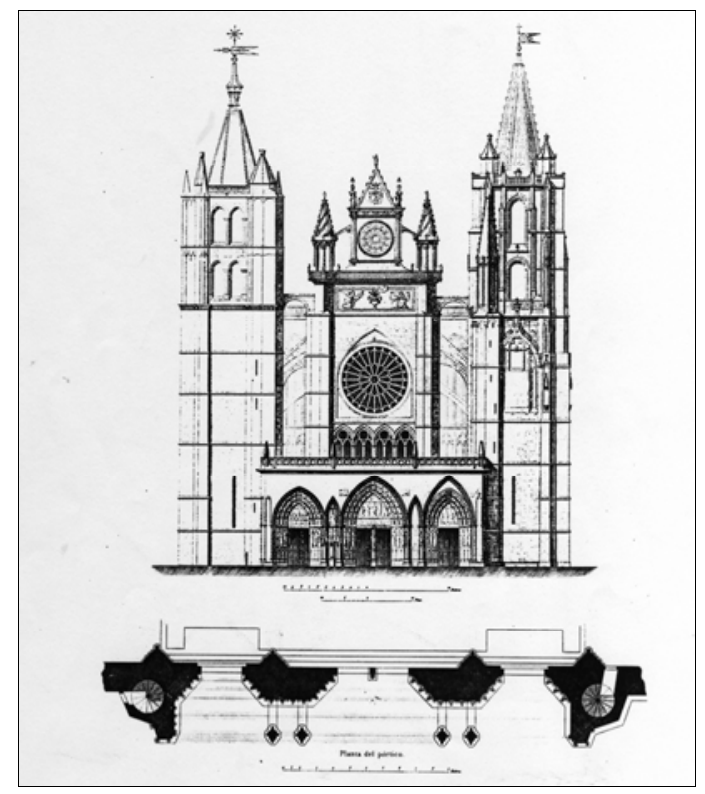

- Fig. 3. Alzado de la fachada occidental de la Catedral de León antes de su desmonte, de Ricardo Velázquez Bosco (1868).

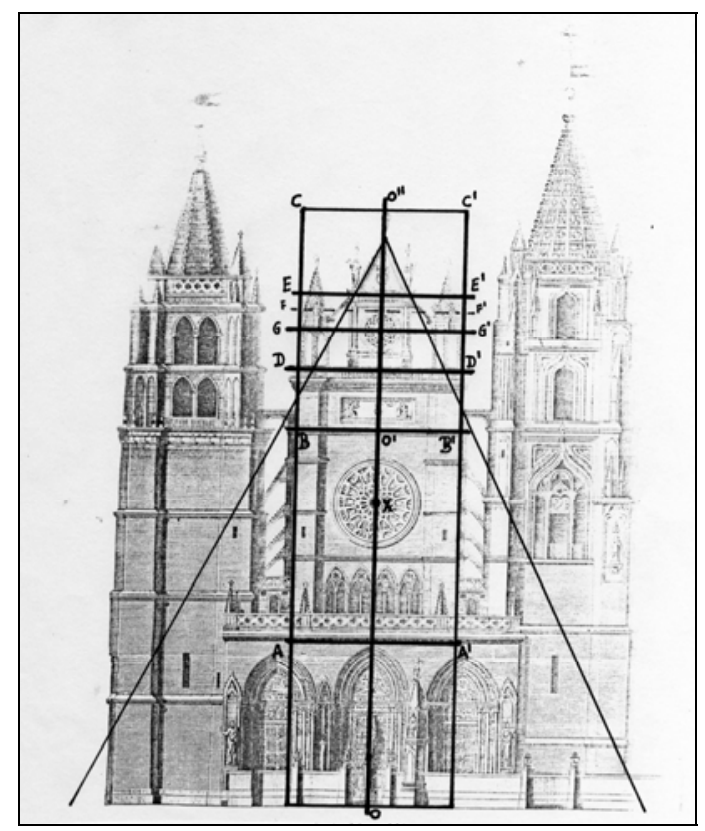

- Fig. 5. Trazado del diseño proporcional del hastial renacentista, sobre el alzado de Manuel Navarro y Fernando Sánchez Pretejo 


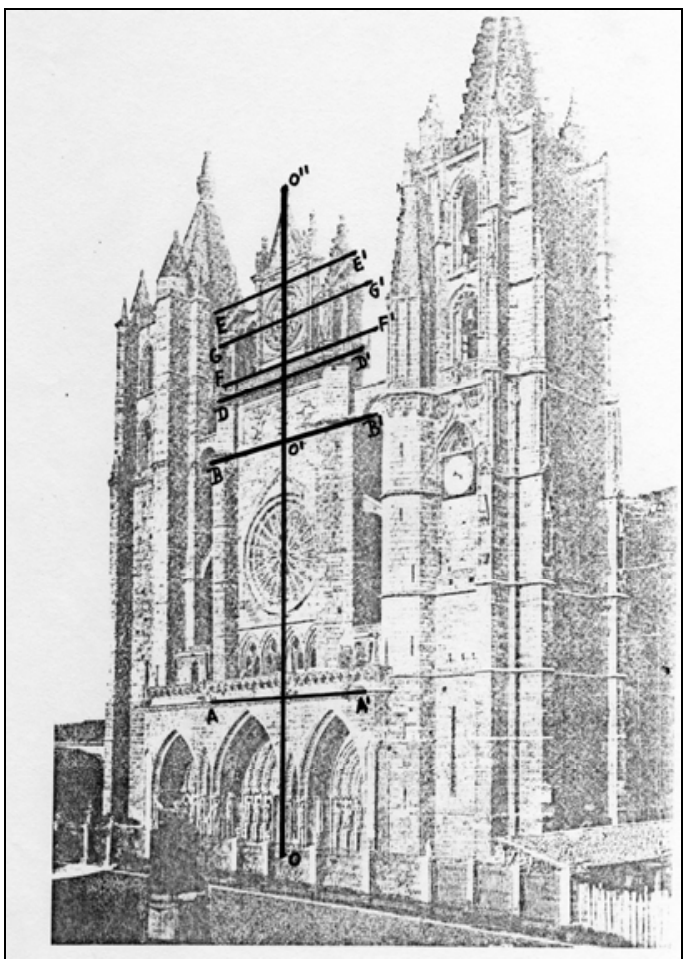

- Fig. 6. Trazado del diseño proporcional del hastial renacentista, sobre la fotografía 1.

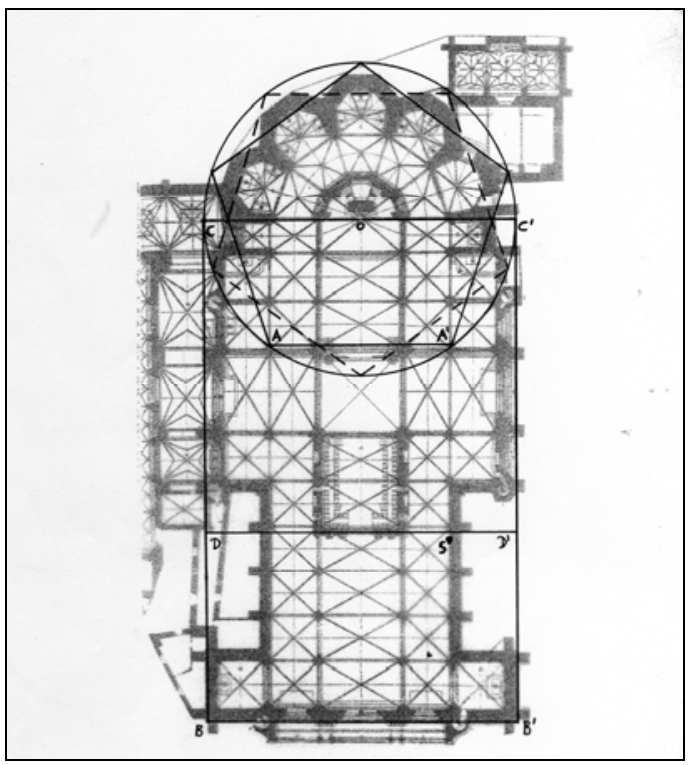

- Fig. 8. Trama geométrica de la Catedral de León, según José Luis Merino de Cáceres, superpuesta a la planta trazada por Demetrio de los Ríos.

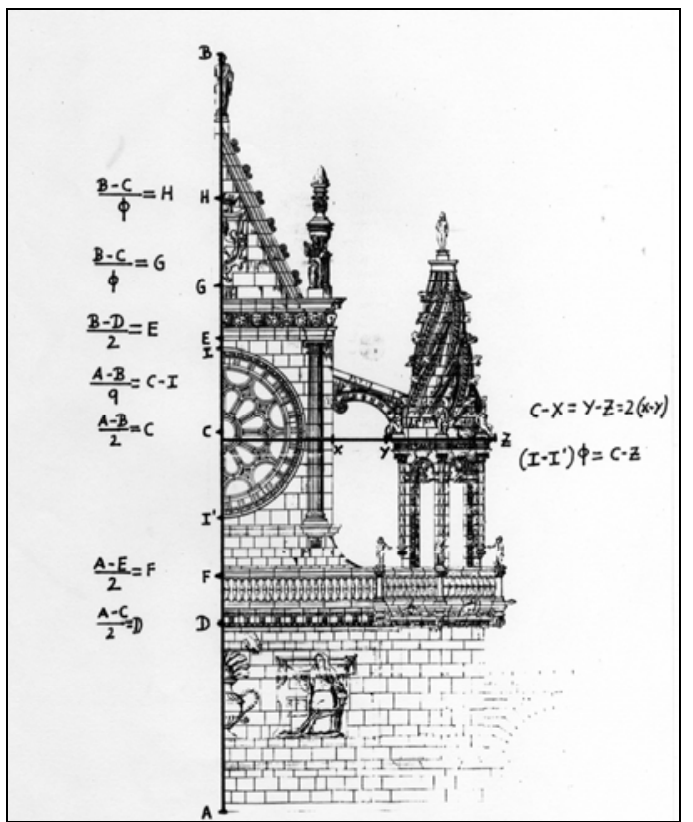

- Fig. 7. Proporciones del hastial renacentista, trazadas sobre el dibujo de Demetrio de los Ríos (1889).

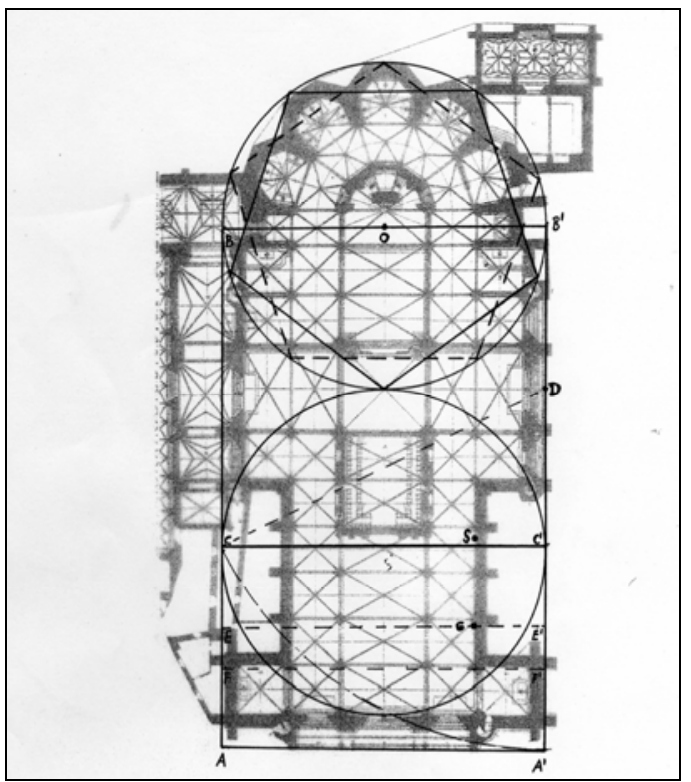

- Fig. 9. Propuesta de César García Álvarez de superposición, corrección y armonización de las propuestas planimétricas de José Luis Merino de Cáceres y Gerardo Boto Varela sobre el trazado de la planta gótica de la Catedral de León. 